\title{
Rejuvenated Frozen Red Blood Cells
}

National Cancer Institute

\section{Source}

National Cancer Institute. Rejuvenated Frozen Red Blood Cells. NCI Thesaurus. Code C133353.

Red blood cells treated with a rejuvenating solution, then frozen and stored at ultra-low temperatures in the presence of a cryoprotective agent. 